TITLE:

\title{
Pseudogap of color superconductivity in heated quark matter
}

\section{$\operatorname{AUTHOR}(\mathrm{S})$ :}

Kitazawa, M; Koide, T; Kunihiro, T; Nemoto, Y

\section{CITATION:}

Kitazawa, M ...[et al]. Pseudogap of color superconductivity in heated quark matter. PHYSICAL REVIEW D 2004, 70(5): 056003.

\section{ISSUE DATE:}

2004-09

URL:

http://hdl.handle.net/2433/50052

RIGHT:

Copyright 2004 American Physical Society 
PHYSICAL REVIEW D, VOLUME 70, 056003

\title{
Pseudogap of color superconductivity in heated quark matter
}

\author{
M. Kitazawa, ${ }^{1}$ T. Koide, ${ }^{2}$ T. Kunihiro, ${ }^{2}$ and Y. Nemoto ${ }^{3}$ \\ ${ }^{1}$ Department of Physics, Kyoto University, Kyoto 606-8502, Japan \\ ${ }^{2}$ Yukawa Institute for Theoretical Physics, Kyoto University, Kyoto 606-8502, Japan \\ ${ }^{3}$ RIKEN BNL Research Center, BNL, Upton, New York 11973, USA
}

(Received 3 September 2003; published 14 September 2004)

\begin{abstract}
We show that the pseudogap of the quark density of states is formed in hot quark matter as a precursory phenomenon of the color superconductivity on the basis of a low-energy effective theory. We clarify that the decaying process of quarks near Fermi surface to a hole and the diquark soft mode $(\mathrm{qq})_{\text {soft }}$ is responsible for the formation of the pseudogap. Our result suggests that the pseudogap is a universal phenomenon in strong coupling superconductors.
\end{abstract}

DOI: 10.1103/PhysRevD.70.056003

PACS numbers: 25.75.Nq, 11.15.Ex, 12.38.Aw

It is an intriguing problem to examine how rich is the phase structure of the high-density $(\rho)$ QCD matter at vanishing or moderate temperature $(T)$. It is now believed that quark matter at an extremely high density undergoes a Cooper instability leading to the color superconductivity (CS) [1-4]: As might be ensured by the asymptoticfree nature of QCD, the quark matter at extremely high densities is usually treated as a Fermi liquid, and the mean-field (MF) theoretical approach à la BCS theory is employed; see, for reviews on recent exciting development, [5].

Various complications are, however, expected on the nature of quark matter at intermediate baryon densities or chemical potential $\mu$ where the strong coupling nature of QCD may show up and invalidate the MF approximation [2]: The strong coupling may make the so-called Ginzburg region so wide that precursory fluctuations of the quark-pair field can have a prominent strength and may give rise to physically significant effects above the critical temperature $\left(T_{c}\right)$ [6]. In this paper we will show that the fluctuation effects of the CS can be so significant that such quark matter may share some basic properties with the cuprates of the high- $T_{c}$ superconductivity (HTSC), having a non-Fermi liquid nature above $T_{c}$.

One of the typical non-Fermi liquid properties of the cuprates is the existence of the pseudogap, i.e., an anomalous depression of density of states (DOS) $N(\omega)$ as a function of the Fermion energy $\omega$ around the Fermi surface above $T_{c}$. Although the mechanism of the pseudogap associated with the HTSC is still controversial, precursory fluctuations of the pair field and the quasitwo-dimensionality of the system seem to be basic ingredients to realize the pseudogap $[7,8]$. In this work, we shall show that the pseudogap of the quark density of states exists as a precursory phenomenon of the CS in a considerable range of $T$ above $T_{c}$ even in the threedimensional system. We calculate the quark self-energy incorporating the preforming pair field of the CS for the first time in the T-matrix approximation (T-approximation $[7,9,10])$, where the amplitude fluctuations are assumed to be dominant over the phase fluctuations of the pair field $[8,11]$. Our result shows that the pseudogap may appear also in a relativistic system solely owing to the amplitude fluctuations, and suggests that the pseudogap phenomenon is universal in strong coupling superconductors, irrespective of the dimensionality as suggested from the study of nuclear matter [9]. Our results should also provide an insight into the physics of proto-neutron stars and heavy-ion collisions as well, where the $\rho$ is relatively low and the effect of finite $T$ plays an important role.

To describe a system at relatively low $T$ and $\rho$, it is appropriate to adopt a low-energy effective theory of QCD $[3,12,13]$. Here we employ a simplified version of the instanton-induced interaction in the two-flavor case known as the Nambu-Jona-Lasinio model $[14,15]$ with the scalar-diquark interaction in the chiral limit,

$$
\begin{aligned}
\mathcal{L}= & \bar{\psi} i \not \partial \psi+G_{C} \sum_{A}\left(\bar{\psi} i \gamma_{5} \tau_{2} \lambda_{A} \psi^{C}\right)\left(\bar{\psi}^{C} i \gamma_{5} \tau_{2} \lambda_{A} \psi\right) \\
& +G_{S}\left[(\bar{\psi} \psi)^{2}+\left(\bar{\psi} i \gamma_{5} \vec{\tau} \psi\right)^{2}\right],
\end{aligned}
$$

where $\psi^{C}(\mathbf{x}) \equiv C \bar{\psi}^{T}(\mathbf{x})$, with $C=i \gamma_{2} \gamma_{0}$ being the charge conjugation operator. Here, $\tau_{2}$ and $\lambda_{A}$ mean the antisymmetric flavor $\mathrm{SU}(2)$ and color $\mathrm{SU}(3)$ matrices, respectively. The coupling $G_{S}$ and the three-dimensional momentum cutoff $\Lambda=650 \mathrm{MeV}$ are determined so as to reproduce the pion decay constant $f_{\pi}=93 \mathrm{MeV}$ and the chiral condensate $\langle\bar{\psi} \psi\rangle=(-250 \mathrm{MeV})^{3}$ in the chiral limit [13]. We choose $G_{C}$ so as to reproduce the phase structure calculated using the instanton-induced interaction [12], i.e., $G_{C}=3.11 \mathrm{GeV}^{-2}$ [13]. We neglect the gluon degrees of freedom, especially their fluctuation, which is known to make the CS phase transition first order in the weak coupling region $[2,16]$; notice that the $\mathrm{CS}$ is a type I in this regime. However, as is emphasized in Ref. [17], nothing definite is known on the characteristics of the CS in the intermediate density region. In this work, simply assuming that the fluctuations of the pair field dominates that of the gluon field as is the case for type II color superconductors, we examine the effects of the 
precursory fluctuations of the diquark-pair field on the quark sector in the T-approximation.

The DOS $N(\omega)$ is calculated from the spectral function $\mathcal{A}(\boldsymbol{k}, \omega)$ of a single quark, which is defined through the spectral representation of the retarded Green function of the quark field:

$$
G^{R}(\boldsymbol{k}, \omega)=\int d \omega^{\prime} \frac{\mathcal{A}\left(\boldsymbol{k}, \omega^{\prime}\right)}{\omega-\omega^{\prime}+i \eta},
$$

and accordingly, $\quad \mathcal{A}(\boldsymbol{k}, \omega)=-1 / \pi \cdot \operatorname{Im} G^{R}(\boldsymbol{k}, \omega) \equiv$ $-1 / \pi \cdot\left(G^{R}-\gamma^{0} G^{R \dagger} \gamma^{0}\right) / 2 i$. From the rotational and parity invariances, the spectral function has the following matrix structure: $\mathcal{A}(\boldsymbol{k}, \omega)=\rho_{0}(\boldsymbol{k}, \omega) \gamma^{0}-\rho_{\mathrm{v}}(\boldsymbol{k}, \omega) \hat{\boldsymbol{k}}$. $\boldsymbol{\gamma}+\rho_{\mathrm{s}}(\boldsymbol{k}, \omega)$, where $\hat{\boldsymbol{k}}=\boldsymbol{k} /|\boldsymbol{k}|$ and $\rho_{\alpha}(\alpha=0, \mathrm{v}, \mathrm{s})$ still have color and flavor indices. Since the quark number is given by $N=\int d^{3} \mathbf{x}\left\langle\bar{\psi} \gamma^{0} \psi\right\rangle$, the DOS is solely given by $\rho_{0}(\boldsymbol{k}, \omega)$,

$$
N(\omega)=4 \int \frac{d^{3} \boldsymbol{k}}{(2 \pi)^{3}} \operatorname{Tr}_{\mathrm{c}, \mathrm{f}}\left[\rho_{0}(\boldsymbol{k}, \omega)\right]
$$

with $\operatorname{Tr}_{\mathrm{c}, \mathrm{f}}$ denoting the trace over color and flavor indices.

The $G^{R}$ in Eq. (2) is given by the analytic continuation of the imaginary-time (Matsubara) Green function $G$, which obeys the following Dyson-Schwinger equation:

$$
\mathcal{G}\left(\boldsymbol{k}, \omega_{n}\right)=\mathcal{G}_{0}\left(\boldsymbol{k}, \omega_{n}\right)\left\{1+\tilde{\Sigma}\left(\boldsymbol{k}, \omega_{n}\right) \mathcal{G}\left(\boldsymbol{k}, \omega_{n}\right)\right\},
$$

where $G_{0}\left(\boldsymbol{k}, \omega_{n}\right)$ and $\tilde{\Sigma}\left(\boldsymbol{k}, \omega_{n}\right)$ denote the free Green function and the self-energy in the imaginary time, respectively. In the normal phase, $\mathcal{G}_{0}$ is reduced to $\mathcal{G}_{0}\left(\boldsymbol{k}, \omega_{n}\right)=\left[\left(i \omega_{n}+\mu\right) \gamma^{0}-\boldsymbol{k} \cdot \boldsymbol{\gamma}\right]^{-1}$ with the Matsubara frequency $\omega_{n}=(2 n+1) \pi T$ for fermions.

As was shown in [6], the fluctuating diquark-pair field develops a collective mode (the soft mode of the CS) at $T$ above but in the vicinity of $T_{c}$, in accordance with the Thouless criterion [18]. Our point in this work is that the soft mode in turn contributes to the self-energy of the quark field, thereby it can modify the DOS so much to give rise to a pseudogap. The quark self-energy $\tilde{\Sigma}$ owing to the soft mode may be obtained by the infinite series of the ring diagrams shown in Fig. 1:

$$
\begin{aligned}
& \tilde{\Sigma}\left(\boldsymbol{k}, \omega_{n}\right)= T \sum_{n_{1}} \int \frac{d^{3} \boldsymbol{k}_{1}}{(2 \pi)^{3}} \tilde{\Xi}\left(\boldsymbol{k}+\boldsymbol{k}_{1}, \omega_{n}+\omega_{n_{1}}\right) \\
& \times G_{0}\left(\boldsymbol{k}_{1}, \omega_{n_{1}}\right), \\
& \tilde{\Xi}\left(\boldsymbol{k}, \nu_{n}\right)=-8 G_{C}\left[1+G_{C} \mathcal{Q}\left(\boldsymbol{k}, \nu_{n}\right)\right]^{-1},
\end{aligned}
$$

with the lowest particle-particle correlation function $\mathcal{Q}\left(\boldsymbol{k}, \nu_{n}\right)$ [6] and $\nu_{n}=2 n \pi T$ being the Matsubara frequency for bosons. Notice that the thin quark lines in Fig. 1 are the free Green function, so we have taken the so-called non-self-consistent approximation, on which we shall make a comment later.

Inserting Eqs. (5) and (6) into Eq. (4) and performing the analytic continuation to the upper half of the complex energy plane, we obtain the retarded Green function, $G^{R}(\boldsymbol{k}, \omega)=\left[G_{0}^{-1}(\boldsymbol{k}, \omega+i \eta)-\Sigma^{R}(\boldsymbol{k}, \omega)\right]^{-1}$, with

$$
\begin{aligned}
\Sigma^{R}\left(\boldsymbol{p}, p^{0}\right)= & \int \frac{d^{3} \boldsymbol{q}}{(2 \pi)^{3}} \int \frac{d \omega}{2 \pi} \frac{-\operatorname{Im} \Xi^{R}(\boldsymbol{p}+\boldsymbol{q}, \omega)}{\omega-p^{0}-E_{\boldsymbol{q}}+\mu-i \eta} \\
& \times \frac{E_{\boldsymbol{q}} \gamma^{0}-\boldsymbol{q} \cdot \boldsymbol{\gamma}}{2 E_{\boldsymbol{q}}}\left[\tanh \frac{E_{\boldsymbol{q}}-\mu}{2 T}-\operatorname{coth} \frac{\omega}{2 T}\right] \\
& +\left(E_{\boldsymbol{q}} \rightarrow-E_{\boldsymbol{q}}\right),
\end{aligned}
$$

where $\quad E_{\boldsymbol{q}}=|\boldsymbol{q}|, \quad \Sigma^{R}(\boldsymbol{k}, \omega)=\left.\tilde{\Sigma}\left(\boldsymbol{k}, \omega_{n}\right)\right|_{i \omega_{n}=\omega+i \eta}, \quad$ and $\Xi^{R}(\boldsymbol{k}, \omega)=\left.\tilde{\Xi}\left(\boldsymbol{k}, \nu_{n}\right)\right|_{i \nu_{n}=\omega+i \eta}$. The matrix structure of the self-energy is the same as the spectral function, $\quad \Sigma^{R}(\boldsymbol{k}, \omega)=\Sigma_{0}(\boldsymbol{k}, \omega) \gamma^{0}-\Sigma_{\mathrm{v}}(\boldsymbol{k}, \omega) \hat{\boldsymbol{k}} \cdot \boldsymbol{\gamma}+$ $\Sigma_{\mathrm{s}}(\boldsymbol{k}, \omega) \equiv \gamma^{0}\left(\Sigma_{-} \Lambda_{-}+\Sigma_{+} \Lambda_{+}\right), \quad$ where $\quad \Lambda_{\mp}=(1 \pm$ $\left.\gamma^{0} \boldsymbol{\gamma} \cdot \hat{\boldsymbol{k}}\right) / 2$ denotes the projection operators onto the positive and negative energy states, and accordingly $\Sigma_{\mp}=$ $\Sigma_{0} \mp \Sigma_{\mathrm{v}}$ represents the self-energies of the particles and antiparticles, respectively; notice that $\Sigma_{\mathrm{s}}(\boldsymbol{k}, \omega)=0$ in the chirally restored phase in the chiral limit. Then it can be shown that $\rho_{0}$ is expressed as a sum of the positive- and negative-energy parts: $\rho_{0}(\boldsymbol{k}, \omega)=-1 / 2 \pi \cdot \sum_{\alpha= \pm} \operatorname{Im} \Sigma_{\alpha} /$ $\left\{R_{\alpha}(\boldsymbol{k}, \omega)^{2}+\left(\operatorname{Im} \Sigma_{\alpha}\right)^{2}\right\}, \quad$ where $\quad R_{ \pm}(\boldsymbol{k}, \omega)=\omega \pm|\boldsymbol{k}|+$ $\mu-\operatorname{Re} \Sigma_{ \pm}(k, \omega)$.

A remark is in order here: The Thouless criterion mentioned above tells us that the denominator of $\Xi^{R}, 1+$ $G_{C} Q(\mathbf{0}, 0)$, vanishes at $T=T_{c}$ because of the selfconsistency condition for the diquark condensate at $T=$ $T_{c}$ [6], where $Q(\boldsymbol{k}, \omega)=\left.\mathcal{Q}\left(\boldsymbol{k}, \nu_{n}\right)\right|_{i \nu_{n}=\omega+i \eta}$.

For the numerical calculation, we employ the following cutoff scheme [14,19]: First we notice that the imaginary part of $Q$ is free from ultraviolet divergences. Therefore we first evaluate the imaginary part exactly without introducing a cutoff, and then use the dispersion relation with the imaginary part just obtained to calculate the real part introducing a three-dimensional cutoff $\Lambda$. The imaginary part is nicely found to have the following compact form:

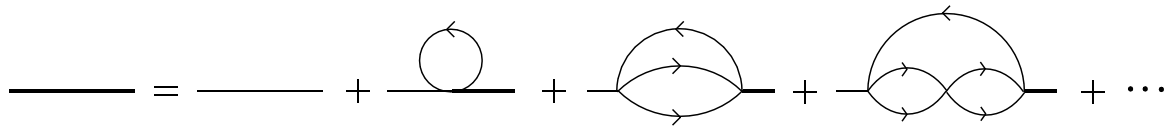

FIG. 1. The Feynman diagrams representing the quark Green function. The thin lines represent the free propagator $G_{0}$, while the bold ones represent the full propagator $G$. 


$$
\begin{gathered}
\operatorname{Im} Q(\boldsymbol{k}, \omega)=-N_{f}\left(N_{c}-1\right) T \frac{(\omega+2 \mu)^{2}-k^{2}}{2 \pi k} \\
\times\left[\theta(|\omega+2 \mu|-k) \ln \frac{\cosh (\omega+k) / 4 T}{\cosh (\omega-k) / 4 T}\right. \\
\left.\quad+\theta(-|\omega+2 \mu|+k) \ln \frac{1+e^{-(\omega+k) / 2 T}}{1+e^{-(-\omega+k) / 2 T}}\right] .
\end{gathered}
$$

We remark that each term has the respective interpretation in terms of the kinetic processes [6]. We emphasize that the compact expression of $\operatorname{Im} Q(\boldsymbol{k}, \boldsymbol{\omega})$ above extremely improves the efficiency of the numerical calculations. Nevertheless, the calculations still involve multiple integrations for obtaining $\Sigma^{R}$ and hence $\rho_{0}$.

Since $\rho_{0}(\boldsymbol{k}, \omega)$ for $\omega>-\mu$ is well approximated solely by the positive-energy part, we first see the characteristic properties of $\Sigma_{-}$. Figure 2 shows a typical behavior of the real and imaginary parts of $\Sigma_{-}$with $k=k_{F}$ at $\mu=$ $400 \mathrm{MeV}$ and the reduced temperature $\varepsilon \equiv(T-$ $\left.T_{c}\right) / T_{c}=0.01$; we remark that $T_{c}=40.04 \mathrm{MeV}$ in the present case [6]. From the figure, one can see that $\operatorname{Re} \Sigma_{-}$ shows a rapid increase around the Fermi energy $\omega=0$. The quark dispersion relation $\omega=\omega_{-}(k)$ therefore also shows a similar behavior around the Fermi surface; $\omega_{-}(k)$ is the solution of $R_{-}(\boldsymbol{k}, \omega)=0$. Hence the density of states proportional to $\left(\partial \omega_{-} / \partial k\right)^{-1}$ becomes smaller near the Fermi surface, which suggests the existence of a pseudogap, provided that the imaginary part $\operatorname{Im} \Sigma_{-}$is neglected, which will be discussed shortly. One can also see that $\omega_{-}\left(k=k_{F}\right) \simeq k-\mu$ since $\operatorname{Re} \Sigma_{-}$at $\omega=0$ is vanishingly small, which will be found important for the pseudogap formation around the Fermi surface.

A numerical calculation shows that as the momentum $k$ is varied from $k_{F}$, the peak of $\left|\operatorname{Im} \Sigma_{-}\right|$at $\omega \approx 0$ seen in Fig. 2 moves along $\omega \approx-k+\mu$ [20]. This means that the quasiparticles with this energy are dumped modes. Figure 1 tells us that $\operatorname{Im} \Sigma_{-}$describes a decay process of a quark to a hole and a diquark, $\mathrm{q} \rightarrow \mathrm{h}+(\mathrm{qq})$, where the hole is on shell with a free dispersion relation $\omega_{h}=\mu-$ $\left|\mathrm{k}_{h}\right|$. The essential point for the pseudogap formation is that the above process is enhanced when the diquark (qq)

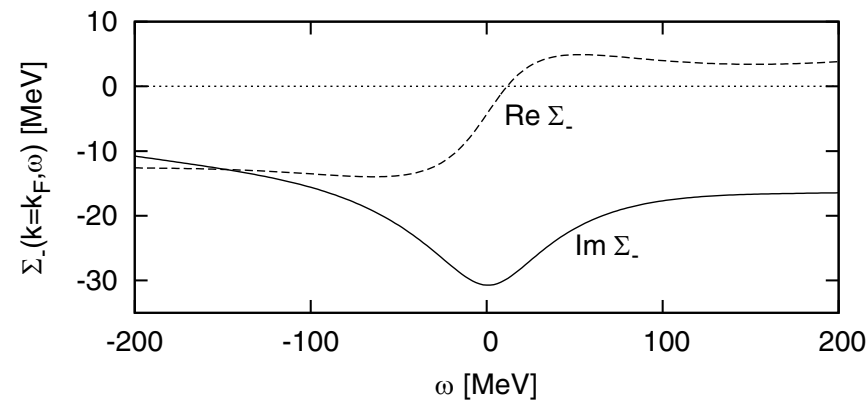

FIG. 2. The self-energy $\Sigma_{-}$with $k=k_{F}$ at $\mu=400 \mathrm{MeV}$ and $\varepsilon \equiv\left(T-T_{c}\right) / T_{c}=0.01$. One observes a peak in $\operatorname{Im} \Sigma_{-}$at $\omega=0$ and a rapid increase of $\operatorname{Re} \Sigma_{-}$at the same $\omega$. makes a collective mode, which we have emphasized is

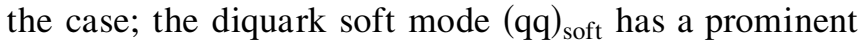
strength at vanishingly small energy $\omega_{s}$ and momentum $\mathrm{k}_{s}$ near $T_{c}$. Owing to the energy-momentum conservation, the energy momentum of the decaying particle $\left(\omega_{p}, \boldsymbol{k}_{p}\right)$ should satisfy $\omega_{p}+\omega_{h}=\omega_{s} \simeq 0$ and $\boldsymbol{k}_{p}+$ $\boldsymbol{k}_{h}=\boldsymbol{k}_{s} \simeq 0$. It means that when the decaying particle has almost the same energy as the free quark, $\left|\operatorname{Im} \Sigma_{-}(\boldsymbol{k}, \omega)\right|$ has the largest value.

The spectral function $\rho_{0}(\boldsymbol{k}, \omega)$ is shown in Fig. 3, at the same $\mu$ and $\varepsilon$ as those in Fig. 2. One can see two families of peaks around $\omega=\omega_{-}(k) \approx k-\mu$ and $\omega=-k-\mu$, which correspond to the quasiparticle peaks of the quarks and antiquarks, respectively. A notable point is that the quasiparticle peak has a clear depression around $\omega=0$, i.e., the Fermi energy. The mechanism for the depression is easily understood in terms of the characteristic properties of the self-energy mentioned above: In fact, $R_{-}\left(k_{F}, \omega \simeq \mu-k_{F}\right) \simeq 0$ and $\left|\operatorname{Im} \Sigma_{-}(\boldsymbol{k}, \omega)\right|$ becomes large when $\omega \approx-k+\mu$. Thus $\rho_{0}\left(k \simeq k_{F}, \omega \simeq 0\right) \simeq$ $-1 /\left[2 \pi \operatorname{Im} \Sigma_{-}\left(k_{F}, \omega \simeq 0\right)\right]$, which is found to be suppressed.

Integrating $\rho_{0}$, one obtains the $\operatorname{DOS} N(\omega)$ : Fig. 4 shows the DOS at $\mu=400 \mathrm{MeV}$ and various values of the reduced temperature $\varepsilon$ together with that of the free quark system, $N_{0}(\omega)$. As anticipated, one can see a remarkable depression of $N(\omega)$, i.e., the pseudogap, around the Fermi energy $\omega=0 ; N(\omega) /\left.N_{0}(\omega)\right|_{\omega=0} \simeq 0.64$ at $\varepsilon=$ 0.01 . One sees that the smaller $\varepsilon$, the more remarkable the rate of depression. The clear pseudogap structure survives even at $\varepsilon=0.05$. One may thus conclude that there is a pseudogap region within the normal phase above $T_{c}$ up to

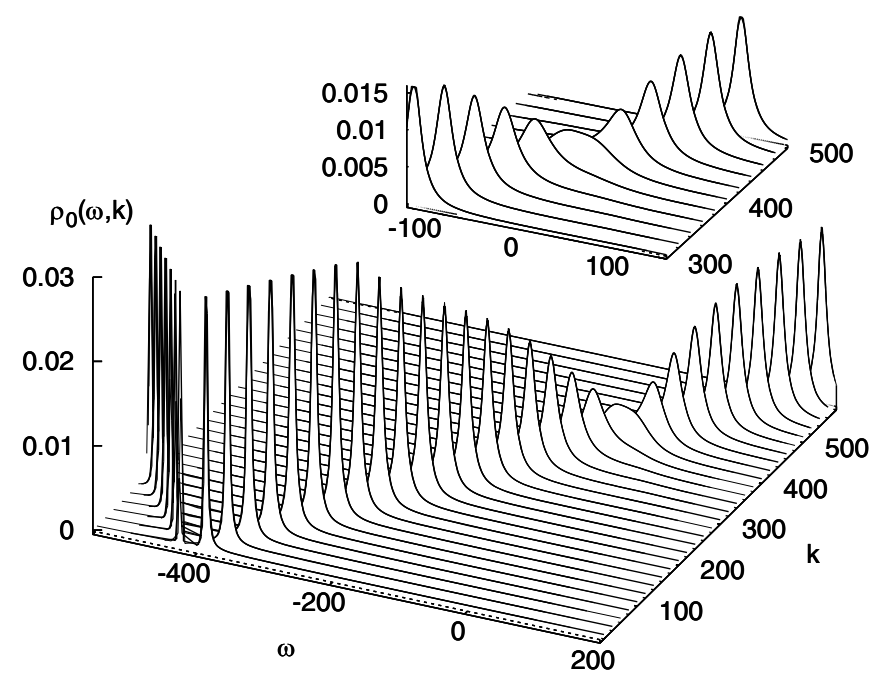

FIG. 3. The spectral function $\rho_{0}$ at $\mu=400 \mathrm{MeV}$ and $\varepsilon=$ 0.01 . The upper figure is an enlargement of the one near the Fermi surface. The peaks at $\omega=k-\mu$ and $\omega=-k-\mu$ correspond to the quark and antiquark quasiparticles, respectively. Notice that there is a depression around $\omega=0$, which is responsible for the pseudogap formation. 


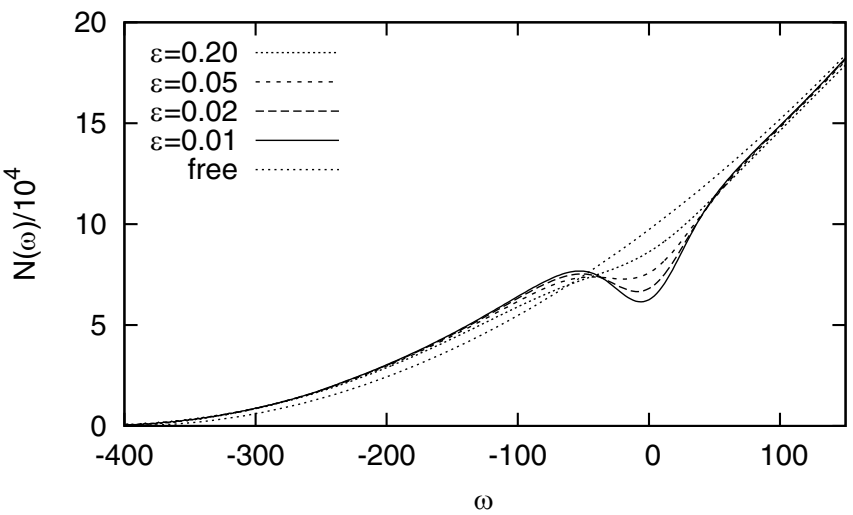

FIG. 4. Density of states at $\mu=400 \mathrm{MeV}$ and various $\varepsilon \equiv$ $\left(T-T_{C}\right) / T_{C}$. The dotted line shows that of the free quarks. A clear pseudogap structure is seen, which survives up to $\varepsilon \approx$ 0.05 .

$T=(1.05-1.1) T_{c}$ at $\mu=400 \mathrm{MeV}$, for instance. A numerical calculation shows that $\varepsilon$ dependence of the width of the pseudogap region hardly changes for $320<\mu<500 \mathrm{MeV}$.

We notice that the pseudogap region obtained in the present work is more than 1 order of magnitude wider in the unit of $\varepsilon$ than in the nuclear matter [9] where the clear pseudogap is seen up to $\varepsilon \approx 0.0025$. This is just a reflection of the strong coupling nature of the QCD at intermediate density region. Our result obtained for a threedimensional system tells us that a considerable pseudogap can be formed without the help of the low dimensionality as in the HTSC and that the pseudogap phenomena in general may be universal in any strong coupling superconductivity.

In this paper we have found that the pseudogap can be formed as a precursory phenomenon of the CS in a rather wide region of $T$ above $T_{c}$. This may imply that quark matter shares some basic properties with the cuprates of the HTSC. It should be noted that our work is the first calculation to show the formation of the pseudogap in the relativistic framework.

In the present work, we have employed the non-selfconsistent T-approximation, while the self-consistent approximation apparently seems better. However, it may not be the case [22]; higher-order terms with the vertex corrections, which are usually discarded in the selfconsistent approximation, cancel with each other, which means that the lowest-order calculation such as ours is more reliable than the self-consistent one [22].

As a future problem, one should consider how to observe the pseudogap in heavy-ion collisions or protoneutron stars. It is also interesting to see what would happen if the phase transition of the CS is strong first order.

We thank K. Iida and D. Rischke for their valuable comments and for drawing our attention to [17,21], respectively. T. Kunihiro is supported by Grant-in-Aide for Scientific Research by Monbu-Kagaku-sho (No. 14540263).
[1] B. C. Barrois, Nucl. Phys. B129, 390 (1977).

[2] D. Bailin and A. Love, Phys. Rep. 107, 325 (1984).

[3] R. Rapp, T. Schafer, E.V. Shuryak, and M. Velkovsky, Phys. Rev. Lett. 81, 53 (1998); M. G. Alford, K. Rajagopal, and F. Wilczek, Nucl. Phys. B537, 443 (1999).

[4] M. Iwasaki and T. Iwado, Prog. Theor. Phys. 94, 1073 (1995); D. T. Son, Phys. Rev. D 59, 094019 (1999); R. D. Pisarski and D. H. Rischke, Phys. Rev. Lett. 83, 37 (1999); T. Schafer and F. Wilczek, Phys. Rev. D 60, 114033 (1999).

[5] K. Rajagopal and F. Wilczek, hep-ph/0011333; M. G. Alford, Annu. Rev. Nucl. Part. Sci. 51, 131 (2001); R. Casalbuoni and G. Nardulli, Rev. Mod. Phys. 76, 263 (2004); J. A. Bowers, hep-ph/0305301.

[6] M. Kitazawa, T. Koide, T. Kunihiro, and Y. Nemoto, Phys. Rev. D 65, 091504(R) (2002).

[7] Y. Yanase and K. Yamada, J. Phys. Soc. Jpn. 70, 1659 (2001).

[8] See, for example, V. M. Loktev, R. M. Quick, and S. G. Sharapov, Phys. Rep. 349, 1 (2001).

[9] A. Schnell, G. Ropke, and P. Schuck, Phys. Rev. Lett. 83, 1926 (1999).
[10] L. P. Kadanoff and G. Baym, Quantum Statistical Mechanics (Benjamin, New York, 1962).

[11] A possible pseudogap formation in association with the chiral transition due to the phase fluctuation was discussed earlier by others: E. Babaev, Int. J. Mod. Phys. A 16, 1175 (2001), in which the possible relevance of the pseudogap to the CS is also mentioned; see also K. Zarembo, JETP Lett. 75, 59 (2002).

[12] J. Berges and K. Rajagopal, Nucl. Phys. B538, 215 (1999).

[13] T. M. Schwarz, S. P. Klevansky, and G. Papp, Phys. Rev. C 60, 055205 (1999).

[14] Y. Nambu and G. Jona-Lasinio, Phys. Rev. 122, 345 (1961); Phys. Rev. 124, 246 (1961).

[15] S. P. Klevansky, Rev. Mod. Phys. 64, 649 (1992); T. Hatsuda and T. Kunihiro, Phys. Rep. 247, 221 (1994).

[16] T. Matsuura, K. Iida, T. Hatsuda, and G. Baym, Phys. Rev. D 69, 074012 (2004).

[17] R. D. Pisarski, Phys. Rev. C 62, 035202 (2000); see also S. Mo, J. Hove, and A. Sudbø, Phys. Rev. B 65, 104501 (2002).

[18] D. J. Thouless, Ann. Phys. (N.Y.) 10, 553 (1960). 
[19] S. Klimt, M. Lutz, U. Vogl, and W. Weise, Nucl. Phys. A516, 429 (1990); U. Vogl, M. Lutz, S. Klimt, and W. Weise, Nucl. Phys. A516, 469 (1990).

[20] The peak structure of the $\left|\operatorname{Im} \Sigma_{-}\right|$around $\omega=0$ is a typical non-Fermi liquid behavior, although the wave function renormalization $Z$ of the quasiparticle at the Fermi surface is found to be $Z=[1+$ $\left.d \operatorname{Re} \Sigma_{-}(\boldsymbol{k}, \omega) / d \omega\right]_{k=k_{F}, \omega=0}^{-1} \approx 0.7$, at $\mu=400 \mathrm{MeV}$ and $\varepsilon=0.01$, which suggets that the non-Fermi liquid picture is not complete; see, for other possible origins of the non-Fermi liquid behavior of quark matter, [21].

[21] D. Boyanovsky and H. J. de Vega, Phys. Rev. D 63, 034016 (2001).

[22] S. Fujimoto, J. Phys. Soc. Jpn. 71, 1230 (2002). 